\title{
A CASE REPORT OF INTRAMUSCULAR LORAZEPAM IN CATATONIA OF A PARKINSON'S DISEASE PATIENT AFTER DEEP BRAIN STIMULATION
}

\author{
Chih-Wei Hsu, Chin-Chuen Lin \& Tiao-Lai Huang \\ Department of Psychiatry, Kaohsiung Chang Gung Memorial Hospital \\ and Chang Gung University College of Medicine, Kaohsiung, Taiwan
}

received: 13.3.2017;

revised: 9.5.2017;

accepted: 2.8.2017

\section{INTRODUCTION}

In Diagnostic and Statistical Manual (DSM)-5, catatonia is defined by the presence of three or more of 12 psychomotor disturbances, which may be associated with mental disorder or medical condition (Tandon et al. 2013). Catatonia could be treated with benzodiazepine (BZD) or electroconvulsive therapy (ECT) (Lin \& Huang 2013, Huang et al. 2013, Raveendranathan et al. 2012). Parkinson's disease (PD) is characterized by motor disturbances that manifests as tremor, rigidity, and bradykinesia. Deep brain stimulation (DBS) is a surgical procedure that implants an electrical pacemaker to deep brain structures, such as subthalamic nucleus and the globus pallidus internus. DBS has been found to be effective in managing motor problem for refractory PD (Lukins et al. 2014). There had been only few reports regarding catatonia in patient with PD (Poyraz et al. 2016) or in patients treated with DBS (Coffey et al. 2010, Quinn et al. 2014). Treatment responses of BZD in those reports were inconsistent. Here, we present a female patient with PD treated with deep brain stimulation (DBS) for 18 months who suddenly developed catatonia during hospitalization. Literature regarding catatonia associated with PD and DBS was reviewed.

\section{CASE REPORT}

This case had been mentioned briefly in another paper discussing relapses and recurrences of catatonia (Lin et al. 2016). However, given the rarity of such a case, we found a more detailed case report is warranted. The patient gave informed consent.

Mrs. Y, a 59-year-old female was diagnosed with PD with initial presentation of bradykinesia, rigidity, and resting tremor at the age of 46 . She was treated with levodopa (100 mg/day) and carbidopa (25 mg/day) at first, but the dosages were increased with levodopa (1600 mg/day) and carbidopa (400 mg/day) in addition to entacapone ( $800 \mathrm{mg} /$ day) in the next decade. During this period, she had two major depressive episodes manifested with depressive mood, decreased appetite, insomnia, feelings of worthlessness, and suicide at- tempts by drug overdose, which resulted in two psychiatric hospitalizations, at age 47 and 56. Antidepressants, including paroxetine (20 to $40 \mathrm{mg} /$ day) and citalopram (20 to $30 \mathrm{mg} /$ day), were used for her two major depressive episodes, respectively. Because her symptoms of PD were deteriorated over time despite high dosage of anti-Parkinson medications, bilateral DBS were implanted at age of 57. After the procedure, her symptoms of PD improved significantly, and she no longer needed oral medications. At age of 58, she was observed to have another major depressive episode with auditory hallucination and persecutory delusion. Under the impression of major depressive disorder with psychotic feature, she was initially prescribed with mirtazapine (60 mg/day), which improved her depressive symptoms, but her psychosis persisted. Olanzapine (10 mg/day) was added, then her psychotic symptoms improved gradually without presence of extrapyramidal syndrome (EPS). Afterward, she was prescribed with olanzapine (5 mg/day) as maintenance treatment.

At age of 59, she was admitted for fluctuating consciousness, disorientation, poor oral intake, auditory hallucination and persecutory delusion, and agitation for 5 days. Her family denied recent history of illicit drug use or head injury. Laboratory data showed hypokalemia (K: $2.7 \mathrm{mEq} / \mathrm{L}$ ) without other electrolyte imbalance, infection, anemia, abnormal renal function, elevation liver enzyme, or metabolic abnormalities. Under the impression of hypokalemia due to poor oral intake for 5 days and then delirium due to hypokalemia (Greenlee et al. 2009), intravenous and oral supplement of potassium were prescribed, and olanzapine (5 $\mathrm{mg} /$ day) was discontinued. Blood potassium was corrected to $3.4 \mathrm{mEq} / \mathrm{L}$ on Day 4 . She became more orientated but remained drowsy, and continued to have auditory hallucination, persecutory delusion, decreased activities with intermittent agitation and rigidity. Anticholinergic drug (trihexyphenidyl $6 \mathrm{mg} /$ day) was added for rigidity, suspected to be EPS, but no improvement was noted from Day 4 to 6 . We then suspected her rigidity was related to DBS, so the neurologist was consulted on Day 6. Upon re-calibration of DBS, her rigidity improved only slightly. Olanzapine (5 mg/day) was added back on 
Day 6 as well, in order to treat her persistent psychotic symptoms. From Day 6 to 10, she remained mostly drowsy, silent, hypoactive, and rigid. However, she could respond to and interact with the staff during the period.

On Day 11, she was noted to have no response to external stimuli, no verbal output, fixed gaze, and kept sitting on a chair with the same, uncomfortable posture. She could not follow order and refused oral intake. According to Bush-Francis Catatonia Rating Scale (BFCRS) (Bush et al. 1996), she presented with stupor, mutism, staring, posturing, rigidity, negativism, and withdrawal, scoring 16 points. Intramuscular (IM) lorazepam (2 mg) challenge improved all catatonic symptoms except residual rigidity after 60 minutes, when her BFCRS score was only one. The patient began to communicate with her caregivers, and started eating. Oral lorazepam (1 mg three times per day) was prescribed. Neurologist was consulted again for residual rigidity but no improvement was found. Another similar episode of catatonia was observed in the wee hours by the doctor on call, and another injection of $2 \mathrm{mg}$ of lorazepam quickly relieved her catatonia. She was discharged smoothly on Day 13 with maintenance olanzapine ( $5 \mathrm{mg} /$ day) and lorazepam ( $3 \mathrm{mg} /$ day). She underwent surgery for DBS adjustment 8 months later, and was free of catatonia during the period.

\section{DISCUSSION}

Catatonia could occur with medical condition, but there are only few reports of catatonia associated with PD or DBS (Tandon et al. 2013, Poyraz et al. 2016, Coffey et al. 2010, Quinn et al. 2014). To our knowledge, two cases following DBS were reported to have catatonia, and one of them also had PD, who developed catatonic symptoms during DBS implantation (Coffey et al. 2010, Quinn et al. 2014). We report the first case of catatonia in a patient with PD treated with DBS for 18 months, then she presented with stupor, mutism, staring, posturing, rigidity, negativism, and withdrawal. The characteristics of the three cases are summarized in Table 1.

In terms of demographic data, all three cases were females around their 60s, and they all presented with decreased activities or consciousness disturbance prior to catatonia. A clinical trial, which enrolls 205 patients, reports predominantly female patients in those with catatonia (37.1\% vs $21.7 \%$ ) and patients with delirium prior to hospitalization (32.3\% vs 16.7\%) (Grover et al. 2014). The three cases show similar result, but the mechanism of phenomenon is unclear.

In the three cases, the numbers of catatonic symptoms of BFCRS are nine, four, and seven, respectively. Stupor, mutism, and negativism were present in all three cases. That finding is consistent with a previous study, which indicates a high prevalence of mutism, stupor, staring, posturing, and negativism in catatonia (Bush et al. 1997). Moreover, all three cases have received DBS, but case 1 (Coffey et al. 2010) and our case had PD.

In terms of treatment response, all three cases improved after lorazepam. We had reported effective management to catatonia in schizophrenia and mood disorder using lorazepam and diazepam (Lin \& Huang 2013, Huang et al. 2013). However, there had been only few reports of treatment of catatonia in neurodegenerative disorder or after brain surgery. Catatonia, PD, and DBS in subthalamic nucleus may be associated with hyperactivity of N-methyl-d-aspartate receptor (NMDAR) in the striato-cortical pathway or the cortico-cortical pathway (Lukins et al. 2014, Inta et al. 2015, Hallett \& Standaert 2004, Akita et al. 2010). Lorazepam as the NMDAR antagonist most likely acts to improve catatonic symptoms by increasing GABA-A and dopamine in aforementioned pathways (Carroll et al. 2007).

\section{CONCLUSIONS}

We report a case of catatonia in a patient with PD after DBS, which was relieved by lorazepam quickly.

Table 1. Summary of Previous 2 Case Reports of Catatonia After DBS

\begin{tabular}{llll}
\hline Variables & Case 1 & Case 2 & Our Case \\
\hline Age & 60 & 67 & 59 \\
Sex & Female & Female & Female \\
PD before DBS & Yes & NA & Yes \\
Time to onset* & During implantation & 6 months after & 18 months after \\
Symptoms of BFCRS & Stupor, mutism, posturing, grimacing, & Stupor, mutism, & Stupor, mutism, staring, \\
& rigidity, negativism, mitgehen, & negativism, withdrawal & posturing, rigidity, \\
& gegenhalten, grasp reflex & & negativism, withdrawal \\
Treatment & Lorazepam (IV) & Lorazepam (IV) & Lorazepam (IM) \\
Time to response** & 4 minutes & Within 30 minutes & 60 minutes \\
Response by BFCRS & 24 to 10 & NA & 16 to 1 \\
\hline
\end{tabular}

Abbreviation: PD: Parkinson's disease; DBS: Deep brain stimulation; NA: Not available;

BFCRS: Bush-Francis catatonia rating scale; IV: Intravenous; ECT: Electroconvulsive therapy; IM: Intramuscular

* Time is calculated from deep brain stimulation implantation to the occurrence of catatonia

**Time is calculated from Lorazepam injection to more than fifty percent response by BFCR 


\section{Acknowledgements:}

This work was supported by grants from Chang Gung Memorial Hospital in Taiwan (Grand number:

CMRPG8C0831) provided to Tiao-Lai Huang.

Conflict of interest: None to declare.

\section{Contribution of individual authors:}

Chih-Wei Hsu searched the literatures and first drafted the manuscript.

Chin-Chuen Lin reviewed the references and revised the manuscript.

Tiao-Lai Huang approved the final version of manuscript. All authors read and approved the final manuscript and contributed to the drafting and revising of the paper.

\section{References}

1. Akita H, Honda Y, Ogata M, Noda K, Saji M: Activation of the NMDA receptor involved in the alleviating aftereffect of repeated stimulation of the subthalamic nucleus on motor deficits in hemiparkinsonian rats. Brain Res 2010; 1306:159-167

2. Bush G, Fink M, Petrides G, Dowling F, Francis A: Catatonia. I. Rating scale and standardized examination. Acta Psychiatr Scand 1996; 93:129-136

3. Bush G, Petrides G, Francis A: Catatonia and other motor syndromes in a chronically hospitalized psychiatric population. Schizophr Res 1997; 27:83-92

4. Carroll BT, Goforth HW, Thomas C, Ahuja N, McDaniel $W W$, Kraus MF et al: Review of adjunctive glutamate antagonist therapy in the treatment of catatonic syndromes. $J$ Neuropsychiatry Clin Neurosci 2007; 19:406-412

5. Coffey MJ, Chou KL, Patil PG: Catatonia during deep brain stimulator implantation complicated by intracranial hemorrhage. Mov Disord 2010; 25:1097-1098
6. Greenlee M, Wingo CS, McDonough AA, Youn JH, Kone $B C$ : Narrative review: evolving concepts in potassium homeostasis and hypokalemia. Ann Intern Med 2009; 150:619-625

7. Grover S, Ghosh A, Ghormode D: Do patients of delirium have catatonic features? An exploratory study. Psychiatry Clin Neurosci 2014; 68:644-651

8. Hallett PJ \& Standaert DG: Rationale for and use of NMDA receptor antagonists in Parkinson's disease. Pharmacol Ther 2004; 102:155-174

9. Huang YC, Lin CC, Hung YY, Huang TL: Rapid relief of catatonia in mood disorder by lorazepam and diazepam. Biomed J 2013; 36:35-39

10. Inta D, Sartorius A, Gass P: NMDA receptor blockade and catatonia: A complex relationship. Schizophr Res 2015; 168:581-582

11. Lin CC \& Huang TL: Lorazepam-diazepam protocol for catatonia in schizophrenia: a 21-case analysis. Compr Psychiatry 2013; 54:1210-1214

12. Lin CC, Hung YY, Tsai MC, Huang TL: Relapses and recurrences of catatonia: 30-case analysis and literature review. Compr Psychiatry 2016; 66:157-165

13. Lukins TR, Tisch S, Jonker B: The latest evidence on target selection in deep brain stimulation for Parkinson's disease. J Clin Neurosci 2014; 21:22-27

14. Poyraz BC, Aksoy Poyraz C, Yassa A, Arikan MK, Gunduz A, Kiziltan G: Recurrent Catatonia in Parkinson Disease. J Clin Psychopharmacol 2016; 36:104-106

15. Quinn DK, Rees C, Brodsky A, Deligtisch A, Evans D, Khafaja $M$ et al: Catatonia after deep brain stimulation successfully treated with lorazepam and right unilateral electroconvulsive therapy: a case report. J ECT 2014; 30:e13-15

16. Raveendranathan D, Narayanaswamy JC, Reddi SV: Response rate of catatonia to electroconvulsive therapy and its clinical correlates. Eur Arch Psychiatry Clin Neurosci 2012; 262:425-430

17. Tandon R, Heckers S, Bustillo J, Barch DM, Gaebel W, Gur RE et al: Catatonia in DSM-5. Schizophr Res 2013: 150:26-30

Correspondence:

Tiao-Lai Huang, MD

Department of Psychiatry, Chang Gung Memorial Hospital-Kaohsiung Medical Center

123, Ta-Pei Rd, Niao-Sung, Kaohsiung 833, Taiwan

E-mail:a540520@adm.cgmh.com.tw 